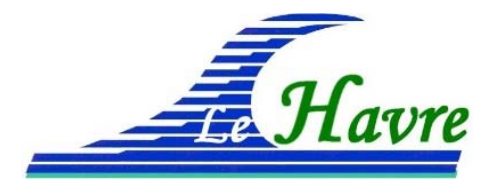

XVI èmes Journées Nationales Génie Côtier - Génie Civil

Le Havre, 2020

DOI:10.5150/jngcgc.2020.033 (C) Editions Paralia CFL

disponible en ligne - http://www.paralia.fr - available online

\title{
Dynamique des barres sédimentaires des plages d'Anglet sous l'effet des rechargements d'avant côte
}

\author{
Didier RIHOUEY ${ }^{1}$, Damien DAILLOUX ${ }^{1}$, Théo BERLAND ${ }^{1}$
}

1. CASAGEC INGENIERIE, 18 rue Maryse Bastié, Zone de Maignon, 64600 Anglet, France.

contact@casagec.fr

\section{Résumé :}

Les plages d'Anglet, qui s'étendent sur environ $4 \mathrm{~km}$ entre l'embouchure de l'Adour et le cap St-Martin, constituent un système fortement anthropisé. La morphologie des plages Sud, entrecoupées d'épis et bordées par des enrochements en haut de plage, est différente des plages ouvertes du Nord. Par ailleurs, depuis la reprise des clapages côtiers en 2010 , environ $2500000 \mathrm{~m}^{3}$ de sable ont été clapés dans la zone d'immersion côtière, au droit des plages d'Anglet.

Cette étude s'appuie sur l'analyse détaillée des levés topo-bathymétriques semestriels acquis sur une durée de 10 ans. Les données ont été traitées à partir de différents outils de manière à extraire automatiquement ou semi-automatiquement la position de la crête des barres internes et externes, leurs éloignements cross-shore, leurs largeurs et leurs profondeurs. L'ensemble de ces informations est confronté aux volumes et aux positions des rechargements d'avant côte.

Les résultats permettent de mettre en avant l'influence des ouvrages et des rechargements sur la morphologie et la dynamique des barres d'avant côte.

Mots-clés : Rechargement d'avant côte, Barres sédimentaires, Anglet

\section{Introduction}

Les rechargements sableux constituent une solution "douce" couramment utilisée pour atténuer les phénomènes d'érosion sur les littoraux sableux exposés (HAMM et al. 2002). Communément mis en œuvre et étudiés aux Pays Bas depuis les années 90 (KROON et al., 1994 ; VAN DUIN et al., 2004 ; RADERMACHER et al., 2018), les rechargements d'avant côte restent marginaux en France à l'exception des plages d'Anglet où ils ont été mis en œuvre de façon systématique entre 1976 et 1990 d'être stoppés puis repris depuis 2010 .

Les plages d'Anglet représentent un système plage-dune incomplet différent des systèmes rencontrés généralement sur le reste de la côte aquitaine (RIHOUEY, 2004). En effet, les aménagements côtiers ont fait disparaître complètement la dune littorale naturelle. De plus, la présence d'épis et l'incidence de la houle n'induisent pas de systèmes "barresbaïnes" de la zone intertidale comme ceux rencontrés plus au Nord. Cependant, des barres internes, interrompues par des chenaux de vidanges souvent perpendiculaires à la côte, 


\section{Thème 2 - Dynamique sédimentaire}

sont présentes sur toute la zone intertidale. De même, des barres externes (subtidales) sont présentes toute l'année mais leur géométrie est plus rectiligne que celles des barres en croissant rencontrées dans les Landes ou en Gironde.

Cet article, qui s'appuie sur l'analyse détaillée des levés topo-bathymétriques semestriels acquis depuis 2010, présente l'analyse de la morphodynamique des barres sédimentaires de plage d'Anglet sous influence des aménagements et des rechargements d'avant côte.

\section{Site d'étude : les plages d'Anglet}

Le littoral d'Anglet (Pyrénées Atlantique) est soumis à un déficit sédimentaire depuis plus d'un siècle qui a entrainé un recul spectaculaire du trait de côte particulièrement au début des années 1970 où le recul de la microfalaise atteignit $10 \mathrm{~m} / \mathrm{an}$. L'impact anthropique n'est plus à démontrer et il a été établi que les facteurs suivants ont aggravé les phénomènes naturels d'érosion : mur de soutènement réfléchissant, construction de la digue Nord du Boucau, dragages du chenal d'accès au port de Bayonne, extractions de granulats sur l'estran.

Entre 1974 et 1979, six épis et une digue en enrochements ont été édifiés pour limiter le recul du trait de côte sur la partie Sud des plages. Parallèlement, entre 1974 et 1990 la majeure partie des sables dragués à l'embouchure de l'Adour pour maintenir l'accès au port de Bayonne ont été clapés au droit des plages Sud. Depuis la réalisation de ces aménagements, le littoral d'Anglet n'a plus subi de dégradation de l'ampleur de celle observée au début des années 1970. L'analyse des levés topo-bathymétriques de 1979 à 2003 montre toutefois un abaissement global des fonds (ABADIE et al., 2004, RIHOUEY, 2004). Cette érosion, "masquée" dans les années 80 sous l'influence des rejets importants de matériaux de dragage au droit des plages Sud, concerne principalement les petits fonds et le bas de l'estran. A contrario, le haut de l'estran et la partie émergée de la plage n'ont pas subi de recul significatif. L'augmentation des pentes de l'estran et les dégradations répétées (2008 et 2009) des musoirs des épis en enrochements ont conduit les collectivités à réaliser des études sur l'érosion des plages et la stabilité des ouvrages (CASAGEC, 2010 ; SOGREAH, 2010).

Face à ces constats, il a été décidé de reprendre les clapages côtiers en 2010 suite à une importante campagne d'analyses physico-chimiques des sédiments pour s'assurer de leur innocuité. En 2015, la CCI Bayonne Pays Basque, en charge des dragages du port de Bayonne, a fait l'acquisition d'une drague à poste pour améliorer les conditions d'accès au port et optimiser les volumes clapés à proximité des côtes. Depuis 2010, environ 2,5 millions de $\mathrm{m}^{3}$ (figure 1) de sable ont été déposés au droit des plages d'Anglet et un suivi topo-bathymétriques biannuels a été mis en œuvre pour évaluer l'efficacité des rechargements d'avant côte. 


\section{XVI èmes Journées Nationales Génie Côtier - Génie Civil \\ Le Havre 2020}

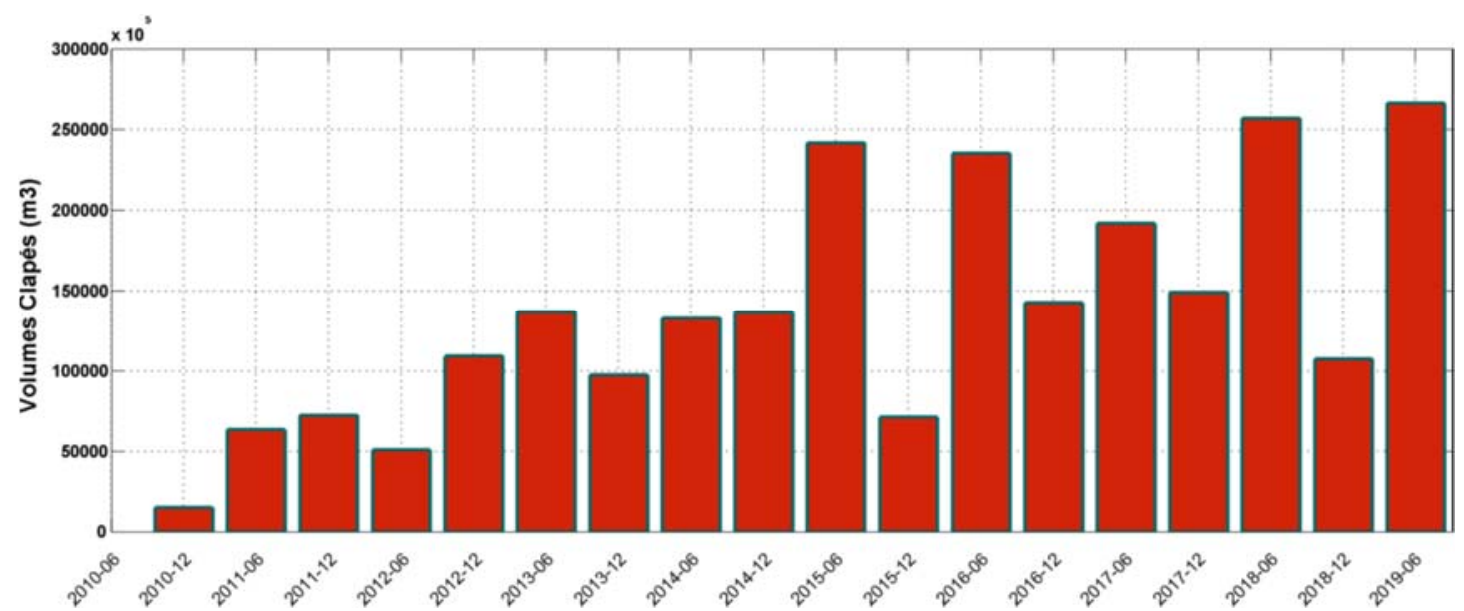

Figure 1. Historique des volumes rechargés au droit des plages depuis 2010.

\section{Données et méthodes}

Les données utilisées dans cette étude consistent en 20 levés topo-bathymétriques réalisés entre 2009 et 2019 avec une fréquence biannuelle (printemps / automne). La zone d'étude s'étend sur 3,7 km de la digue des Cavaliers au Nord au Cap St-Martin au Sud (figure 2a). Les profils bathymétriques, long de $1500 \mathrm{~m}$ et espacés de $100 \mathrm{~m}$, ont été levés à l'aide d'un sondeur mono faisceau. Les données sont interpolées sur une grille régulière avec un pas de $5 \mathrm{~m}$. La carte de la dérivée cross-shore donne un signal visuel direct sur la position des barres (figure 2b). En effet le changement de signe de la dérivée correspond à l'inversion de pente du profil et donne donc la position du creux et de la crête de la barre.
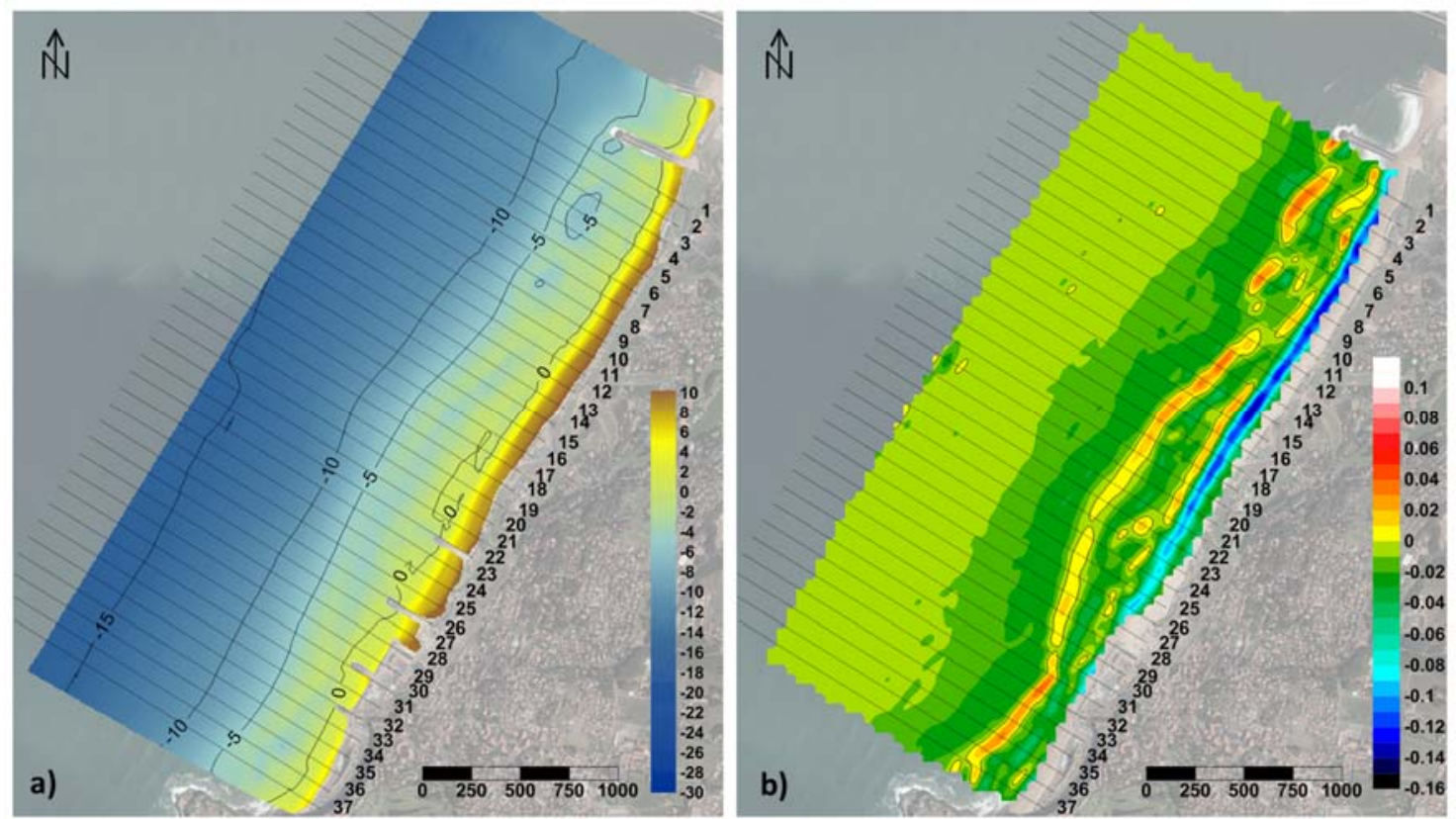

Figure 2. Bathymétrie des plages d'Anglet de mars 2019 (a) et dérivée cross-shore (b). 


\section{Thème 2 - Dynamique sédimentaire}

Un algorithme automatisé a été développé afin de permettre le calcul des paramètres suivants : position du creux et de la crête de la barre, profondeur de la crête de la barre, hauteur de la barre, distance à la côte de la crête de la barre. Sur chaque profil, l'algorithme détecte la première et la deuxième inversion de signe de la dérivée en partant du large. La première inversion de signe correspond à la position de la crête de la barre et la deuxième inversion à la position du creux (figure 3.b). L'intersection entre une droite horizontale en direction du large ayant pour origine la position du creux et le profil bathymétrique permet d'obtenir la largeur de la barre. La hauteur de la barre est obtenue par la différence entre l'altitude du creux et de la crête. L'application de l'algorithme décrit précédemment pour la détection de la barre interne c'est révélé infructueux dans de nombreux cas en raison d'absence d'inversion de signe de la dérivée (figure 3.b). Ainsi, les positions des barres internes ont été digitalisées. Pour limiter les incertitudes liées à la digitalisation, une méthode basée sur la l'utilisation d'une régression polynomiale d'ordre $4 \mathrm{du}$ profil bathymétrique a été développée. En effet, la superposition de polynomiale d'ordre 4 et du profil bathymétrique permet de mettre en évidence la morphologie de la barre (figure 3.a) en l'absence d'inversion de signe de la dérivée.

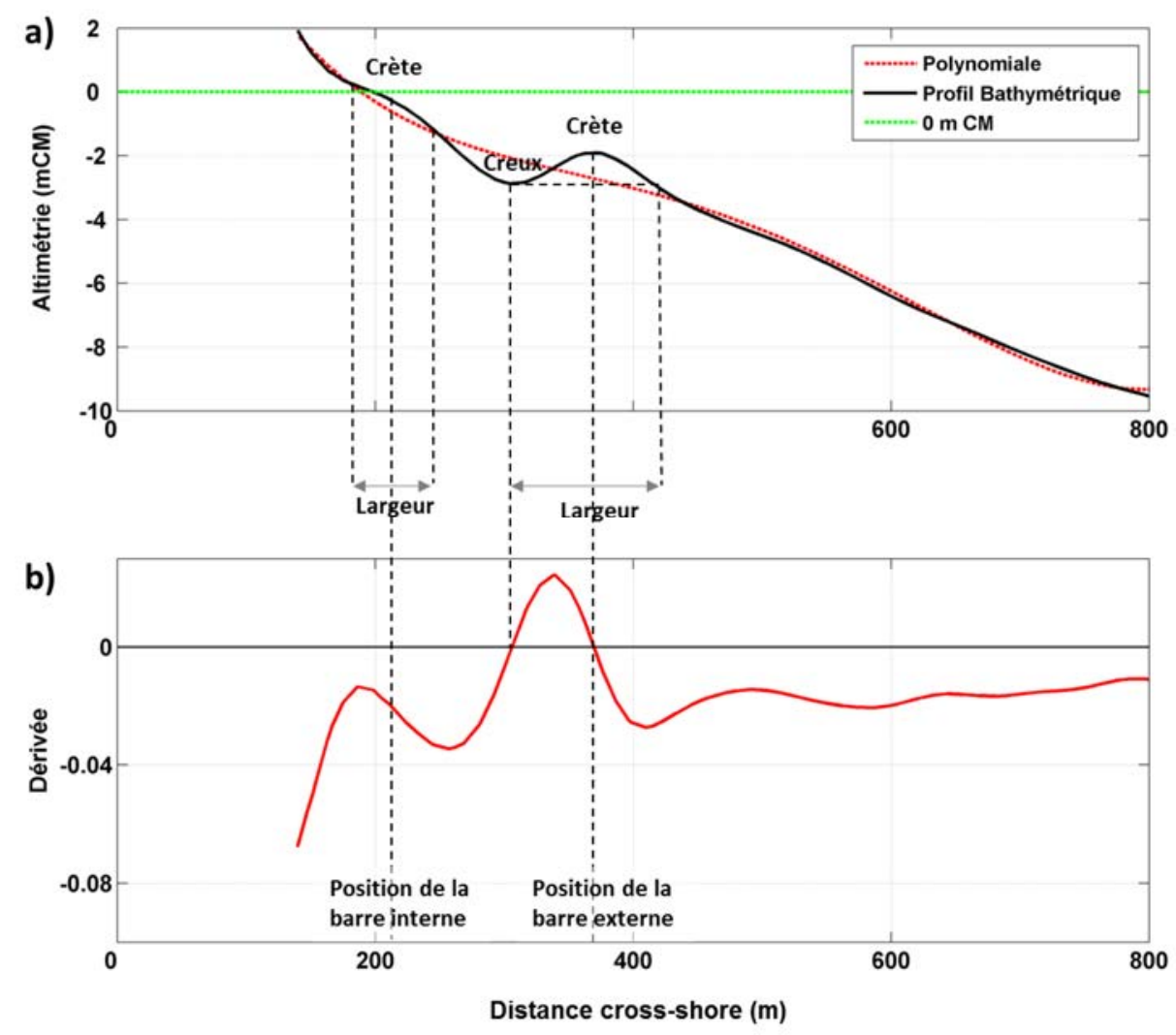

Figure 3. Méthodologies de détection des barres externes basée sur l'inversion de signe de la dérivée (b) et de détection des barres internes basée sur la superposition d'une régression polynomiale d'ordre 4 du profil bathymétrique (a). 


\section{XVI'̀mes Journées Nationales Génie Côtier - Génie Civil \\ Le Havre 2020}

\section{Résultats}

Un grand nombre d'indicateurs de la morphologie des barres internes et externes sont disponibles à la suite du traitement. Ainsi, à la complexité de détection de ces indicateurs, vient s'ajouter la complexité de les représenter dans l'espace et dans le temps. La figure 4 représente l'évolution spatio-temporelle de la morphologie de la barre externe sous forme de points colorés (Altimétrie de la crête de barre) de diamètres variables (largeur de la barre). L'axe des abscisses correspond au numéro de profil et représente donc les plages d'Anglet du Sud (profil 37) au Nord (profil 1). L'axe des ordonnées présente les dates où les levés topo-bathymétriques ont été effectués. Les informations sur les volumes de sédiments clapés sont ajoutées aux graphiques sous la forme de rectangles dont l'emprise correspond à la localisation des rechargements et la largeur correspond aux volumes.

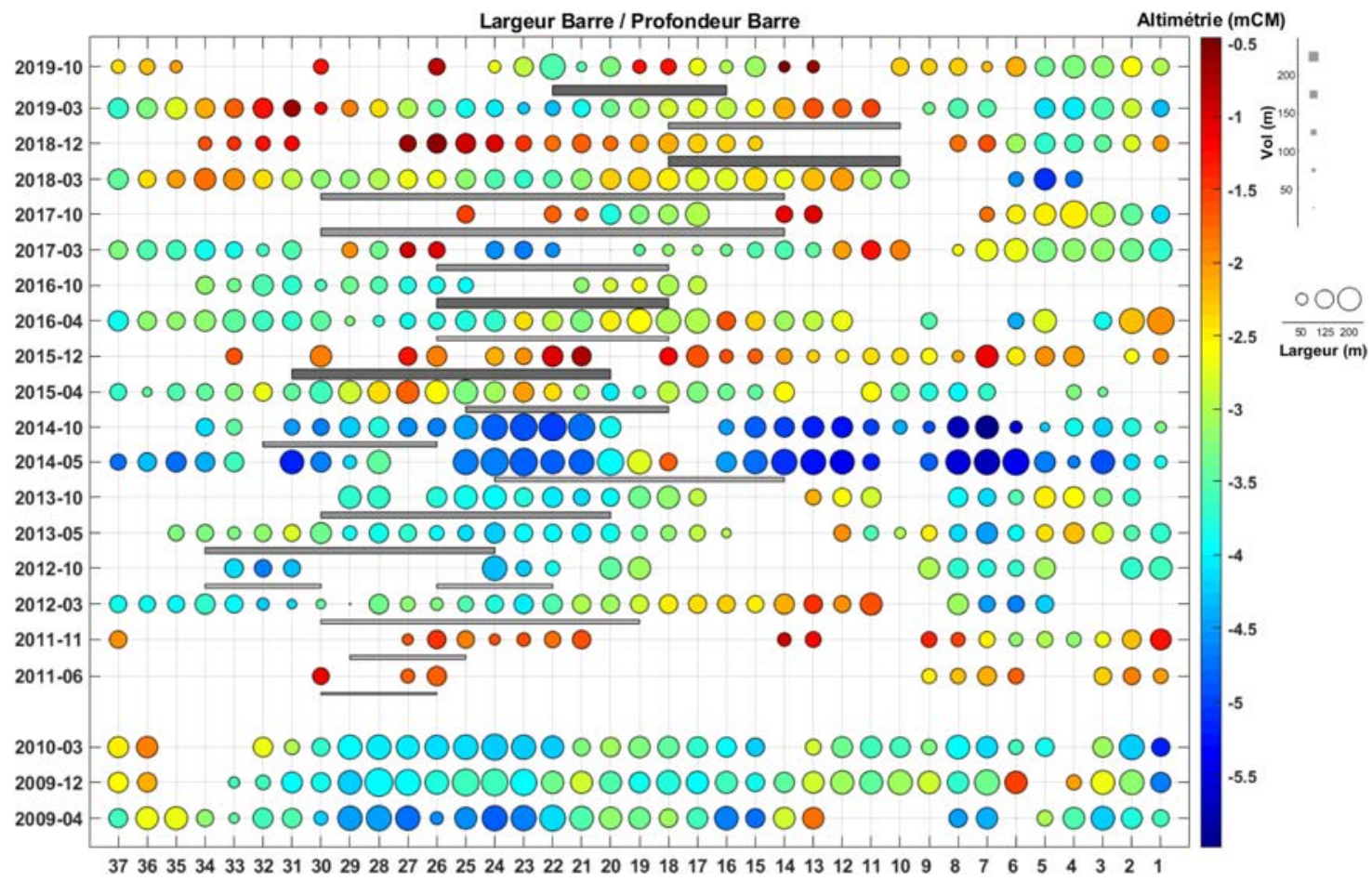

Figure 4. Evolution spatio-temporelle de la largeur (diamètre du cercle) et de la profondeur (couleur) de la barre externe.

L'analyse de l'évolution de la barre externe (figure 4) met en évidence un signal saisonnier avec des barres plus profondes (les couleurs tendent vers le bleu) et plus rectiligne au printemps, et des barres moins profondes (les couleurs tendent vers le rouge) et plus discontinues en automne. L'impact de l'hiver 2013-2014 est clairement identifiable avec un net approfondissement de la barre qui perdure jusqu'à l'automne suivant. L'augmentation des volumes clapés se traduit ensuite par une tendance à la remontée de la hauteur des crêtes majoritairement observable au droit des secteurs 


\section{Thème 2 - Dynamique sédimentaire}

rechargés. Concernant la barre interne, la figure 5 met en évidence une forte variabilité de la profondeur de crête le long des plages. Au Nord (Profil 1 à 15), les barres internes sont relativement profondes (les couleurs tendent vers le bleu) et présentent fréquemment des discontinuités. Sur la partie centrale, caractérisée par la présence d'épis (localisés au niveau des profils $22,26,30,32$ ) et bénéficiant des rechargements d'avant côte, les barres internes sont moins profondes (les couleurs tendent vers le rouge) et relativement continues. Au Sud (Profil 33 à 37) les barres s'approfondissent. En termes d'évolution temporelle, le signal saisonnier et l'impact de l'hiver 2013-2014 sont moins marqués que sur la barre externe.

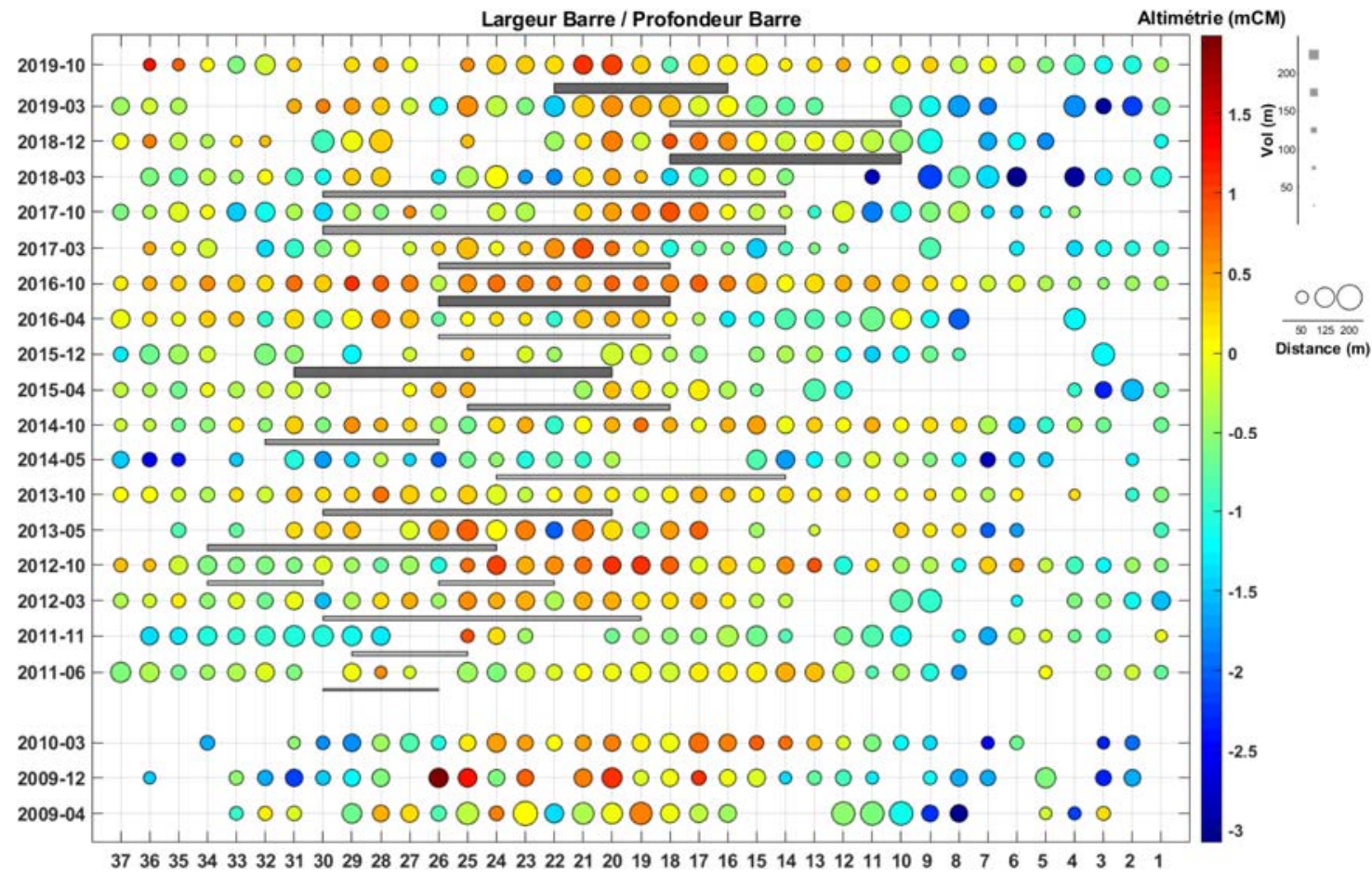

Figure 5. Evolution spatio-temporelle de la largeur (diamètre du cercle) et de la profondeur (couleur) de la barre interne.

L'influence des ouvrages et des rechargements sur la barre interne s'observe nettement sur la figure suivante qui présente les moyennes des profondeurs de crêtes des barres externes (figure 6a) et des barres internes (figure 6b) ainsi que l'écart type associé (la localisation des épis est matérialisée par des rectangles grisés). En effet, les barres internes sont nettement plus stables et moins profondes au niveau des secteurs anthropisés à l'exception des profils dans l'axe des ouvrages où les chenaux de vidange engendrent des discontinuités. A contrario, la profondeur moyenne de la barre externe ne semble pas influencée par la présence des ouvrages. 


\section{XVİ̀mes Journées Nationales Génie Côtier - Génie Civil \\ Le Havre 2020}
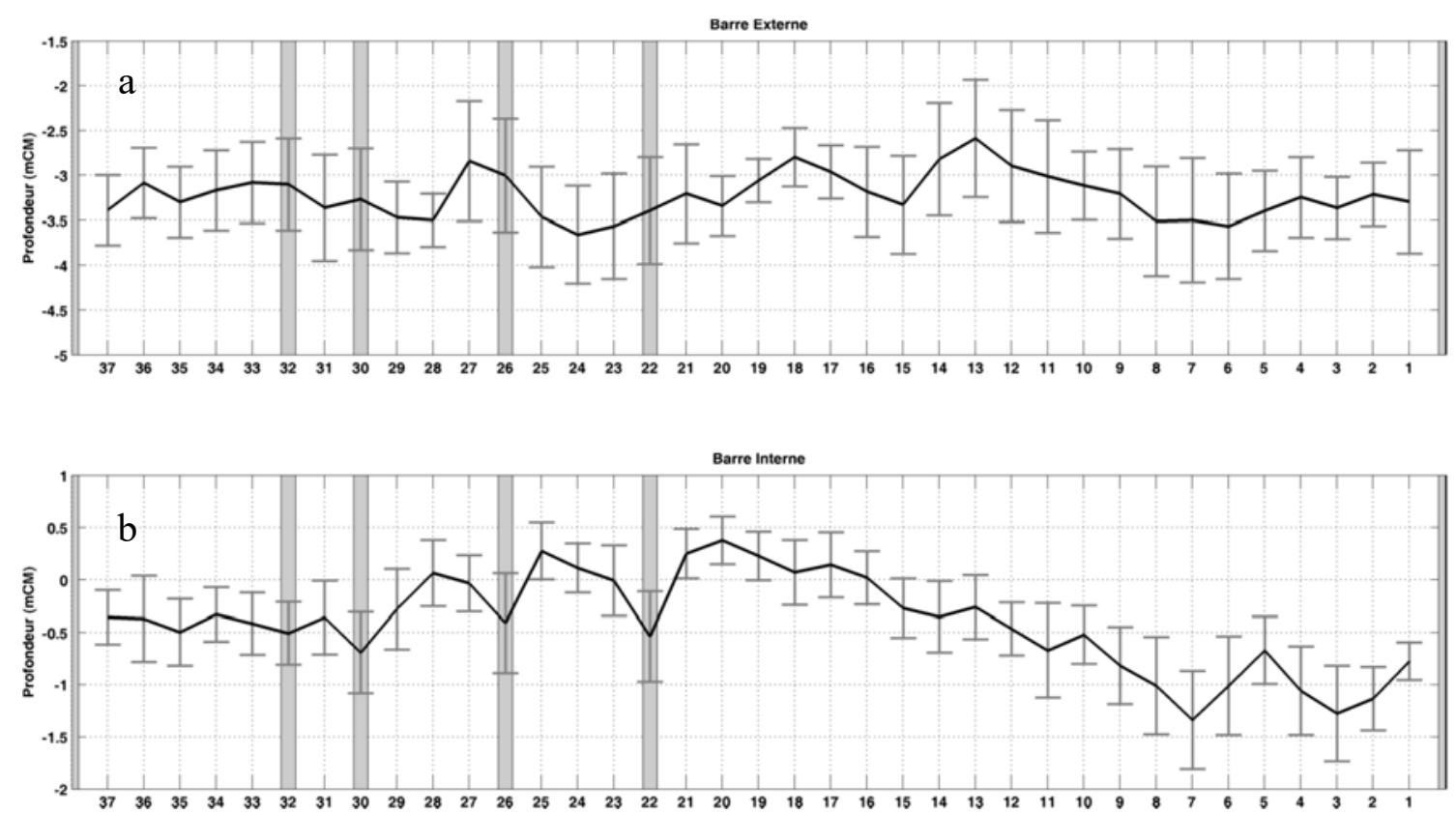

Figure 6. Profondeur moyenne des crêtes des barres externes (a) et interne (b).

\section{Conclusion et discussion}

Une chaîne de traitements de données topo-bathymétriques a été développée pour extraire automatiquement ou semi-automatiquement les caractéristiques des barres internes et externes. Appliquée à 20 levés des plages d'Anglet entre 2009 et 2019 avec une fréquence biannuelle (printemps / automne), cette chaîne de traitement permet d'appréhender la variabilité spatio-temporelle des barres sédimentaires le long des $4 \mathrm{~km}$ du littoral. L'analyse de la barre interne met en évidence une spatialisation très forte en lien avec les aménagements côtiers et les rechargements d'avant côte. Moins profondes et plus continues dans la partie Sud des plages d'Anglet, les barres internes s'apparentent dans la partie Nord aux systèmes barre/baïne aquitains avec une rythmicité importante. Au droit des épis qui segmentent les plages Sud, on note également un approfondissement localisé des barres internes. Enfin, en accord avec la littérature (RADERMACHER et al., 2018), les rechargements de l'avant côte permettent une alimentation des petits fonds observables sur les barres internes. La diminution de la profondeur de la barre interne au droit des secteurs rechargés et la migration vers le Nord de cet effet montre que les rechargements d'avant côte sont efficaces au-delà des zones de clapage.

\section{Références bibliographiques}

ABADIE S., BRIERE C., DUBRANNA J., MARON P., RIHOUEY D. (2004). Analyse de l'évolution du volume sédimentaire des plages subtidales d'Anglet. VIII ${ }^{\text {ème }}$ Journées Nationales Génie Civil - Génie Côtier, Compiègne, 7-9 septembre 2004, pp 711-719. https://doi.org/10.5150/jngcgc.2004.082-A

CASAGEC (2010). Bilan morpho-sédimentaire des plages d'Anglet. Rapport mars 2010. 


\section{Thème 2 - Dynamique sédimentaire}

HAMM L., CAPOBIANCO M., DETTE H., LECHUGA A., SPANHOFF R., STIVE M. (2002). A summary of European experience with shore nourishment. Coast. Eng. 47 (2), pp 237-264. https://doi.org/10.1016/S0378-3839(02)00127-8

KROON A., HOEKSTRA P., HOUWMAN K., RUESSINK G. (1994). Morphological monitoring of a shoreface nourishment NOURTEC Experiment at Terschelling, The Netherlands. Coastal Engineering, Kobe, Japan, 1994, October 23-28, Proceedings ed. B. L. Edge, pp 2223-2236.

RADERMACHER M., DE SCHIPPER, M.A., PRICE T.D., HUISMAN B.J.A., AARNINKHOF S.G.J., RENIERS A.J.H.M. (2018). Behaviour of subtidal sandbars in response to nourishments. Geomorphology, Vol. 313, pp 1-12. https://doi.org/10.1016/j.geomorph.2018.04.005

SOGREAH (2010). Rôle, stabilité et confortement des épis des plages d'Anglet. Rapport $\mathrm{N}^{\circ} 1713024 \mathrm{R} 1$, Juin 2010.

RIHOUEY D. (2004). Analyse statistique de l'évolution morphodynamique des plages sableuses : Application aux sites d'étude du Programme National d'Environnement Côtier et aux plages d'Anglet. Thèse de doctorat de 1'Université de Pau et des Pays de l'Adour.

VAN DUIN M.J.P., WIERSMA N.R., WALSTRA D.J.R., VAN RIJN L.C., STIVE M.J.F. (2004). Nourishing the shoreface: observations and hindcasting of the Egmond case, The Netherlands. Coastal Engineering, Vol. 51(8-9), pp 813-837. https://doi.org/10.1016/j.coastaleng.2004.07.011 